\title{
ÁCIDO ASCÓRBICO EM COUVE FOLHA SOB ESTRESSE SALINO EM REGIÃO SEMIÁRIDA DE ALAGOAS
}

\section{ASCORBIC ACID IN LEAFY CABBAGE UNDER SALT STRESS IN THE SEMI-ARID REGION OF ALAGOAS}

\section{Julianna Catonio da Silva ${ }^{*} \mathbb{D}$, Cinara Bernardo da Silva ${ }^{2}$, Cleice Fátima Gonçalves Alves ${ }^{2}{ }^{\mathbb{D}}$, João Cardoso de Albuquerque Neto ${ }^{3}{ }^{\circledR}$, José Wanderson Silva dos Santos ${ }^{4}{ }^{\circledR}$, José Clebson Barbosa Lúcio5 ${ }^{5}$}

\footnotetext{
${ }^{1}$ Doutoranda em Agronomia, Universidade Federal de Alagoas, Centro de Engenharias e Ciências Agrárias - CECA, Rio Largo, AL, Brasil. ${ }^{2}$ Mestre em Agricultura e Ambiente, Extensionista Emater, Arapiraca, Al, Brasil.

${ }^{3}$ Mestre em Agricultura e Ambiente, Coop. de Trabalho de Profissionais de Agronomia-UNICAMPO, Luís Eduardo Magalhães, BA, Brasil.

${ }^{4}$ Graduando em Agronomia, Universidade Federal de Alagoas, UFAL, Rio Largo, AL, Brasil.

${ }^{5}$ Doutorando em Agronomia, Universidade Estadual Paulista, UNESP, Jaboticabal, SP, Brasil.
}

\begin{abstract}
RESUMO: Objetivou-se estudar os efeitos do ácido ascórbico em couve folha submetida ao estresse salino. O experimento foi conduzido em casa de vegetação, na Universidade Federal de Alagoas - UFAL, Campus de Arapiraca, Arapiraca-AL. Foi adotado um delineamento experimental em blocos casualizados (DBC), com esquema fatorial $5 \times 4$, com cinco repetições. Os tratamentos foram representados por 5 níveis de salinidade $\left(0,12 ; 1,62 ; 3,12 ; 4,62\right.$ e $\left.6,12 \mathrm{dS} \mathrm{m}^{-1}\right)$ e quatro concentrações de ácido ascórbico $(0,5,10$ e $20 \mu \mathrm{M})$. Aos 30 dias após o transplantio foram avaliadas as seguintes variáveis: altura de planta (AP), número de folhas (NF), diâmetro da copa (DC), biomassa fresca da parte aérea (BFPA), área foliar (AF) e a produtividade (PROD). As variáveis: altura de planta, área foliar, biomassa fresca da parte aérea e produtividade apresentaram comportamento linear decrescente ao aumento dos níveis salinos, apresentando redução de: 8,$51 ; 20,52 ; 24,71$ e 22,79\%, respectivamente, do menor ao maior nível salino testado. O aumento das concentrações de ácido ascórbico apresentou efeito positivo para a variável diâmetro da copa, em 8,6\%. Mesmo o ácido ascórbico tendo se mostrado eficiente no aumento do diâmetro da copa, para as variáveis de interesse econômico, não diminuiu os efeitos causados pela salinidade.
\end{abstract}

Palavras-chave: Brassica oleracea L., antioxidantes, salinidade

\begin{abstract}
The objective was to study the effects of ascorbic acid in cabbage leaf submitted to salt stress. The experiment was conducted in a greenhouse at the Universidade Federal de Alagoas - UFAL, Campus de Arapiraca, Arapiraca-AL. An experimental design in randomized blocks (BCT) was adopted, with a 5 x 4 factorial scheme, with five repetitions. The treatments were represented by 5 salinity levels $(0.12 ; 1.62 ; 3.12$; 4.62 and $\left.6.12 \mathrm{dS} \mathrm{m}^{-1}\right)$ and four ascorbic acid concentrations $(0,5,10$ and $20 \mu \mathrm{M})$. At 30 days after transplanting, the following variables were evaluated: plant height (PA), number of leaves (NF), canopy diameter (CD), fresh aboveground biomass (FFB), leaf area (LA) and productivity (PROD). The variables: plant height, leaf area, fresh biomass of the aerial part and productivity presented linear decreasing behavior to the increase of the saline levels, presenting reduction of: $8.51 ; 20.52 ; 24.71$ and $22.79 \%$, respectively, from the lowest to the highest salt level tested. The increase in the concentrations of ascorbic acid showed a positive effect for the variable crown diameter, by $8.6 \%$. Even though ascorbic acid was efficient in increasing the diameter of the crown, for the variables of economic interest, it did not decrease the effects caused by salinity.
\end{abstract}

Key words: Brassica oleracea L., antioxidants, salinity 


\section{INTRODUÇÃO}

A couve folha (Brassica oleracea L.) pertencente à família Brassicaceae é uma hortaliça muita rica em nutrientes, especialmente cálcio, ferro, vitaminas A, C, K, B5 e ácido ascórbico, sendo também uma ótima fonte de carotenoides apresentando, entre as hortaliças, as maiores concentrações de luteína e de beta caroteno (FILGUEIRA, 2013; PEREIRA et al., 2016).

No Brasil, a região mais afetada pelo excesso de sais é o Nordeste, geralmente provocada por irrigação em áreas impróprias (DIAS et al., 2019). As plantas, quando submetidas ao estresse salino, podem apresentar diferentes respostas, sendo fenômeno extremamente complexo, envolvendo alterações morfológicas e de crescimento, além de processos fisiológicos e bioquímicos.

Existem estudos afim de reduzir os efeitos dos sais nas plantas, sendo o ácido ascórbico (AsA), um dos antioxidantes mais importantes na proteção de plantas contra $o$ estresse oxidativo gerado por estresse abiótico (LÚCIO et al., 2018). A aplicação de ácido ascórbico como antioxidante endógeno em plantas é necessária para neutralizar o estresse oxidativo, além de outros processos do metabolismo vegetal.

O Ácido Ascórbico desempenha papel chave na tolerância ao sal de muitas plantas halófitas e é prontamente absorvido após a aplicação exógena movendo-se dentro da planta, sendo a aplicação foliar de AsA capaz de melhorar a tolerância das plantas aos sais (ATHAR; KHAN; ASHRAF; 2008; HAMEED et al. 2015).

Neste sentido, objetivou-se estudar os efeitos da aplicação de ácido ascórbico em couve folha submetida ao estresse salino.

\section{MATERIAL E MÉTODOS}

$\mathrm{O}$ experimento foi desenvolvido em casa de vegetação, em área experimental pertencente a Universidade Federal de Alagoas (UFAL), Campus de Arapiraca, de julho a agosto de 2018, localizada na mesorregião Agreste do Estado de Alagoas, nas coordenadas $9^{\circ} 45^{\prime} 09^{\prime \prime} \mathrm{S}$ e $36^{\circ} 39^{\prime} 40^{\prime \prime} \mathrm{O}$, em altitude de $325 \mathrm{~m}$. Essa região é de transição entre a Zona da Mata e o Sertão alagoano, com clima do tipo 'As' tropical com estação seca de verão, pelo critério de classificação de Köppen (1948).

$\mathrm{O}$ delineamento experimental utilizado foi em blocos casualizados (DBC), num esquema fatorial $5 \times 4$, com 5 repetições. Os tratamentos resultaram da combinação de dois fatores: salinidade da água de irrigação (CEa) em cinco níveis $(0,12 ; 1,62 ; 3,12 ; 4,62$ e 6,12 $\mathrm{dS} \mathrm{m}^{-1}$ ) e quatro concentrações de ácido ascórbico $(0,5,10$ e $20 \mu \mathrm{M})$. A unidade experimental foi representada por um recipiente de polietileno com capacidade para $5 \mathrm{~L}$.

Foram utilizados baldes plásticos com capacidade para 5L. Os recipientes foram preenchidos, inicialmente com uma camada de brita e areia para facilitar a drenagem da água e evitar a passagem de possíveis sedimentos. Em seguida, o recipiente foi preenchido com o solo da área experimental, cuja classificação é LATOSSOLO VERMELHO-AMARELO Distrófico (EMBRAPA, 2018), da camada referente aos $20 \mathrm{~cm}$ iniciais.

Após o preenchimento com o solo, o mesmo foi saturado e colocado em capacidade de campo $(\mathrm{Cc})$ durante 24 horas, em seguida foi liberada a drenagem do excesso de água. A adubação de fundação com Nitrogênio $(\mathrm{N})$, Fósforo (P) e Potássio (K) foi realizada seguindo o manual de recomendação para o 
estado de Pernambuco e a análise de solo da área experimental (Tabela 1). Logo após a adubação, foi realizado o transplantio da cultura da couve folha

Tabela 1. Características químicas e físicas do solo da área experimental

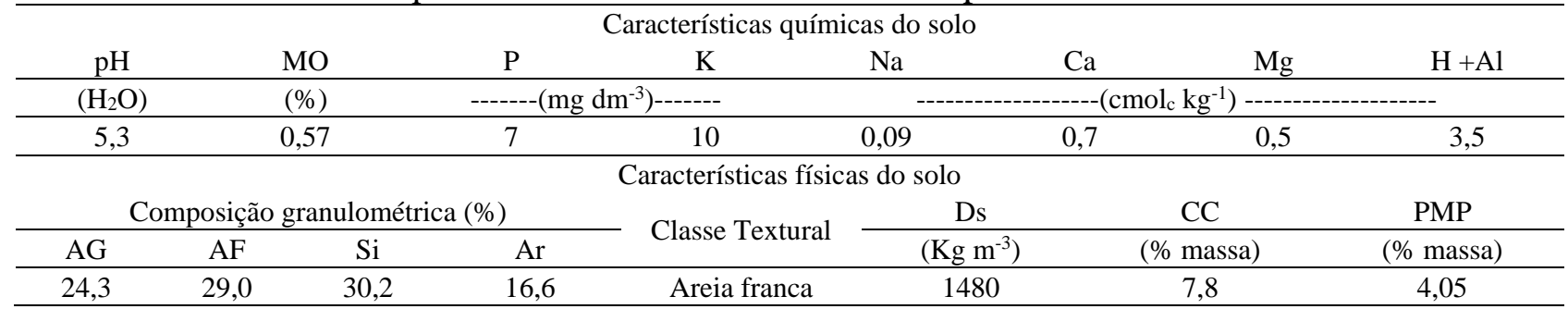

$\mathrm{MO}$ - matéria orgânica; $\mathrm{AG}$ - área grossa; $\mathrm{AF}$ - areia fina; $\mathrm{Si}$ - silte; $\mathrm{Ar}$ - argila; Ds - densidade do solo; $\mathrm{CC}$ - capacidade de campo; PMP - ponto de murcha permanente.

Para o preparo dos tratamentos salinos, multiplicou-se a condutividade desejada por 640 para obter a quantidade de Cloreto de Sódio $(\mathrm{NaCl})\left(\mathrm{mg} \mathrm{L}^{-1}\right)$ necessário para cada nível salino, conforme (RICHARDS, 1954). Entretanto, para o cálculo, foi considerada a concentração de sais já existente na água do abastecimento local $\left(0,12 \mathrm{dS} \mathrm{m}^{-1}\right)$.

A lâmina diária de irrigação foi calculada diariamente através da coleta de um conjunto de 5 lisímetros de drenagem, por meio da diferença entre a lâmina aplicada e a lâmina drenada, sendo utilizada a média dos três valores centrais, desprezando os valores extremos (maior e o menor).

As aplicações das concentrações do AsA foram realizadas a cada 48 horas em todas as folhas de cada planta de acordo com os tratamentos.

Aos 30 dias após o transplantio (DAT) foram avaliadas as seguintes variáveis: altura de planta $(\mathrm{cm})$, número de folhas $(\mathrm{NF})$, diâmetro da copa (DC), biomassa fresca da parte aérea (BFPA), area foliar (AF) e produtividade (PROD).

Os dados foram submetidos à análise de variância pelo teste "F" e de regressão, utilizando o software estatístico SISVAR (FERREIRA, 2011).

\section{RESULTADOS E DISCUSSÃO}

Pela análise da variância realizada, para os níveis de salinidade $\left(\mathrm{dS} \quad \mathrm{m}^{-1}\right)$ e concentrações de ácido ascórbico $(\mu \mathrm{M})$ (Tabela 2), observa-se que a interação desses fatores não apresentou diferença significativa. $\mathrm{O}$ efeito dos níveis de salinidade mostrou-se significativo para a altura da planta (AP), a biomassa fresca da parte aérea (BFPA), área foliar (AF) e a produtividade (PROD), ao nível de $0,01 \%$ de probabilidade. Já o diâmetro da copa (DCOPA) apresentou diferença significativa para as concentrações de ácido ascórbico, ao nível de 0,05\%.

Tabela 2. Análise de variância para altura de planta (AP), número de folhas (F), diâmetro da copa (DC), biomassa fresca da parte aérea (BFPA), área foliar (AF) e a produtividade (PROD)

\begin{tabular}{|c|c|c|c|c|c|c|c|}
\hline \multirow{2}{*}{ Fonte de variação } & \multirow{2}{*}{ GL } & \multicolumn{6}{|c|}{ Quadrados médios } \\
\hline & & $\mathrm{AP}$ & $\mathrm{NF}$ & $\mathrm{DC}$ & BFPA & $\mathrm{AF}$ & PROD \\
\hline Salinidade $(\mathrm{S})$ & 4 & $16,8^{*}$ & $0,2^{\mathrm{ns}}$ & $18,9^{\text {ns }}$ & $910,1 * *$ & $239455,1 * *$ & $0,9 * *$ \\
\hline Ácido ascórbico (AsA) & 3 & $7,3^{\mathrm{ns}}$ & $1,2^{\mathrm{ns}}$ & $43,8^{*}$ & $43,9^{\text {ns }}$ & $40123,1^{\mathrm{ns}}$ & $0,1^{\mathrm{ns}}$ \\
\hline $\mathrm{S} \times \mathrm{AsA}$ & 12 & $8,9^{\text {ns }}$ & $1,4^{\mathrm{ns}}$ & $18,2^{\mathrm{ns}}$ & $86,4^{\mathrm{ns}}$ & $35757,1^{\mathrm{ns}}$ & $0,1^{\mathrm{ns}}$ \\
\hline Bloco & 4 & $8,7^{\mathrm{ns}}$ & $3,7^{\mathrm{ns}}$ & $44,6^{\mathrm{ns}}$ & $710,9^{\text {ns }}$ & $139924,1^{\mathrm{ns}}$ & $0,7^{\mathrm{ns}}$ \\
\hline Erro & 76 & 6,6 & 1,8 & 13,7 & 100,4 & 33682,2 & 0,1 \\
\hline Total & 99 & & & & & & \\
\hline $\mathrm{CV}(\%)$ & & 10,3 & 14,8 & 9,9 & 16,4 & 17,3 & 16,4 \\
\hline
\end{tabular}

$\mathrm{GL}=$ grau de liberdade; $\mathrm{CV}=$ coeficiente de variação; $(*)$ significativo a $5 \%,(* *)$ a $1 \%$ de probabilidade e ${ }^{\text {(ns) }}$ não significativo. 
O aumento da salinidade da água de irrigação apresentou efeito negativo sobre a altura das plantas, com modelo linear decrescente (Figura 1). Observaram-se menores valores quando as plantas foram submetidas a $6,12 \mathrm{dS} \mathrm{m}^{-1}$ da água de irrigação $(23,96 \mathrm{~cm})$, correspondendo a uma redução de $8,51 \%$ em relação ao controle $(26,19 \mathrm{~cm})$. Um efeito comum da salinidade nas plantas é a redução no crescimento já que o excesso de sais no ambiente radicular provoca menor disponibilidade e absorção de água pelas raízes aumentando, consequentemente, a pressão osmótica que provoca inibição na expansão e divisão celular, comprometendo a absorção de nutrientes (MAHAJAN; TUTEJA, 2005).

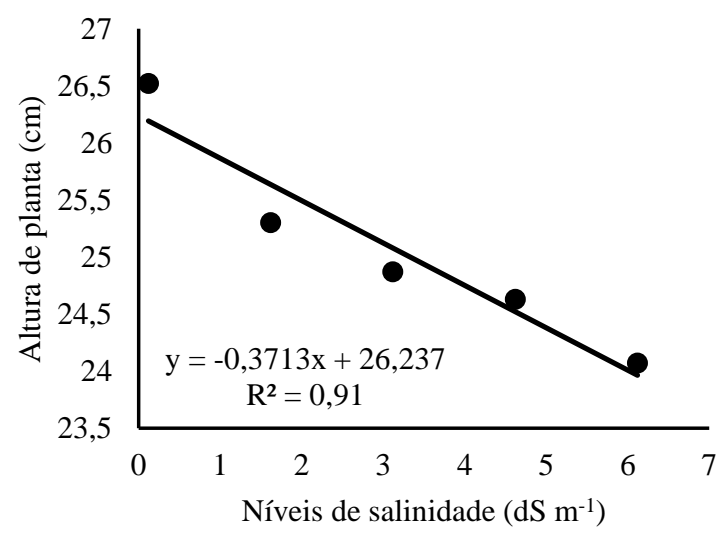

Figura 1. Altura de planta da couve folha $(\mathrm{cm})$ em função de níveis de salinidade $\left(\mathrm{dS} \mathrm{m} \mathrm{m}^{-1}\right)$.

Lima et al. (2015) estudando a cultura da beringela, também observou decréscimo em altura de plantas com o aumento do estresse salino. Da mesma forma, Silva et al. (2017) estudando a cultura da alface, evidenciaram reduções com o aumento da irrigação com água salina.

O aumento das concentrações de ácido ascórbico apresentou efeito positivo para a variável diâmetro da copa (Figura 2). Foram observados menores valores quando o mesmo não foi aplicado $(35,93 \mathrm{~cm})$, correspondendo a uma redução de $8,6 \%$ em relação a concentração de $20(\mu \mathrm{M})(39,02 \mathrm{~cm})$.

$\mathrm{O}$ aumento do diâmetro da copa pode estar relacionado com a formação endógena de hormônios reguladores do crescimento, como a giberelina e citocinina, os quais promovem a elongação e divisão celular (TAIZ; ZEIGER, 2017). Embora seja amplamente conhecido o papel do ácido ascórbico (Asa) na desintoxicação de espécies reativas de oxigênio (EROS), principalmente quando em condições de estresse, salino ou hídrico, atuando como cofator em reações enzimáticas, envolvendo enzimas como a ascorbato peroxidase e glutationa redutase (GALLIE, 2013), o mesmo também está associado à produção de hormônios vegetais que agem no crescimento da planta, como a citocinina e gibirelina (EL-BAZ et al., 2016; DOS SANTOS et. al., 2018).

Em couve flor sob déficit hídrico, que se assemelha aos efeitos do estresse salino, foi observado aumento no conteúdo de clorofilas, potássio, fósforo, prolina e giberelina, ocasionando um aumento no crescimento da planta (MUKHTAR et al., 2016; AKRAM et. al., 2017).

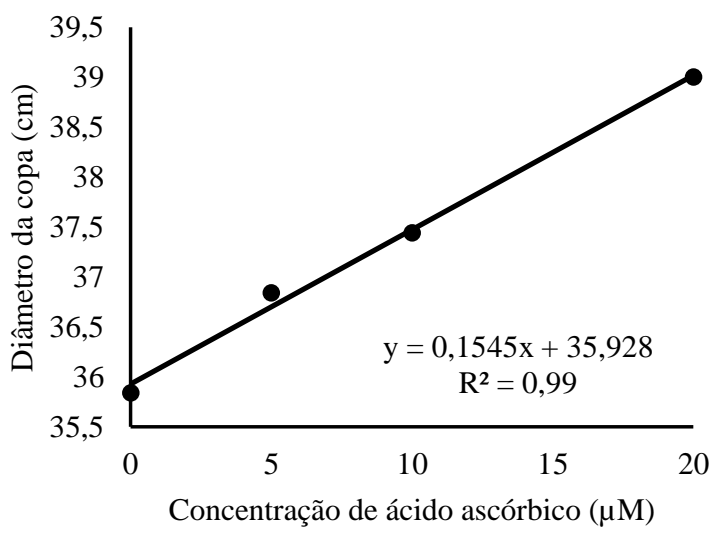

Figura 2. Diâmetro da copa $(\mathrm{cm})$ da couve folha em função de concentrações de ácido ascórbico $(\mu \mathrm{M})$.

Foi verificado maior área foliar, 1178,73 $\mathrm{cm}^{2}$, para o tratamento controle $\left(0,12 \mathrm{dS} \mathrm{m}^{-1}\right) \mathrm{e}$ menor valor, $936,79 \mathrm{~cm}^{2}$, para o tratamento com $6,12 \mathrm{dS} \mathrm{m}^{-1}$ da água de irrigação, havendo assim um decréscimo de 20,52\% (Figura 3). A área foliar apresenta grande relevância para a cultura da couve folha, por ser uma variável de crescimento indicativas da produtividade, visto que, o processo fotossintético depende da interceptação da energia luminosa e sua conversão em energia química, sendo este um processo que ocorre 
diretamente na folha (SILVA et al., 2013; DIAS et al., 2019).

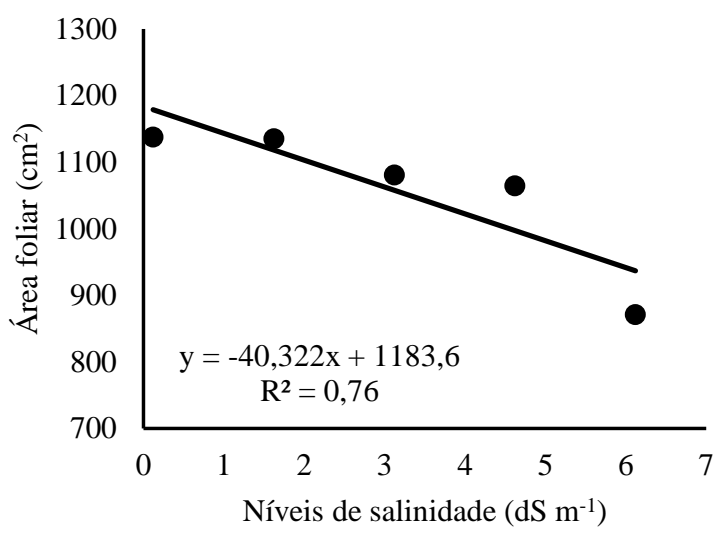

Figura 3. Área foliar $\left(\mathrm{cm}^{2}\right)$ da couve folha em função de níveis de salinidade $\left(\mathrm{dS} \mathrm{m} \mathrm{m}^{-1}\right)$.

A redução da área foliar é um mecanismo adaptativo de plantas cultivadas em condições de excesso de sais e estresse hídrico, visto que, sob tais condições é interessante a redução na transpiração e, consequentemente, diminuição do carregamento de íons $\mathrm{Na}+\mathrm{e}^{-}$no xilema e concomitante conservação de água nos tecidos das plantas. Desta forma, a inibição na expansão do limbo foliar é uma das características mais comuns em plantas submetidas ao estresse, sendo que em algumas culturas já é observado, como na cultura da rúcula (OLIVEIRA et al., 2012), e em outras hortaliças folhosas, como alface (DIAS et al., 2011; SILVA et al., 2017).

O acréscimo dos níveis salinos da água de irrigação apresentou efeito negativo sobre a biomassa fresca da parte aérea e produtividade de couve folha (Figura 4 e 5). Para a biomassa fresca, os menores valores foram encontrados quando as plantas foram submetidas a $6,12 \mathrm{dS}$ $\mathrm{m}^{-1}$ da água de irrigação (52,92 $\left.\mathrm{g} \mathrm{planta}^{-1}\right)$, correspondendo a uma redução de $24,71 \%$ em relação ao controle $\left(70,29 \mathrm{~g}\right.$ planta $\left.^{-1}\right)$.

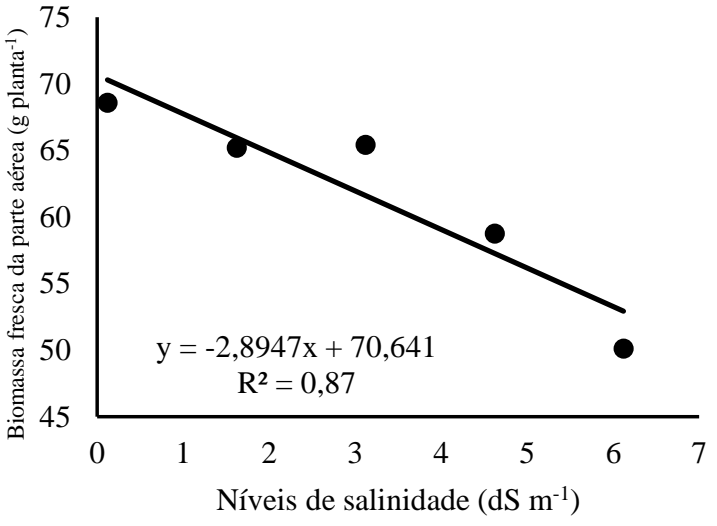

Figura 4. Biomassa fresca da parte aérea $\left(\mathrm{g} \mathrm{planta}^{-1}\right) \mathrm{da}$ couve folha em função de níveis de salinidade $\left(\mathrm{dS} \mathrm{m}^{-1}\right)$.

A maior produtividade $\left(2,15 \mathrm{t} \mathrm{ha}^{-1}\right)$ foi encontrada no tratamento controle e a menor $\left(1,66 \mathrm{t} \mathrm{ha}^{-1}\right)$ no nível de $6,12 \mathrm{dS} \mathrm{m}^{-1}$, ocasionando uma redução de $22,79 \%$ da produtividade (Figura 5).

A redução da produtividade da couvefolha em resposta ao aumento das concentrações de sais da água de irrigação está relacionada à sua tolerância à salinidade, uma vez que essa cultura é classificada como moderadamente sensível, onde apresenta salinidade limiar de $1,8 \mathrm{dS} \mathrm{m}-1$, com decréscimo relativo na produtividade de $9,7 \%$. (Viana, 2017).

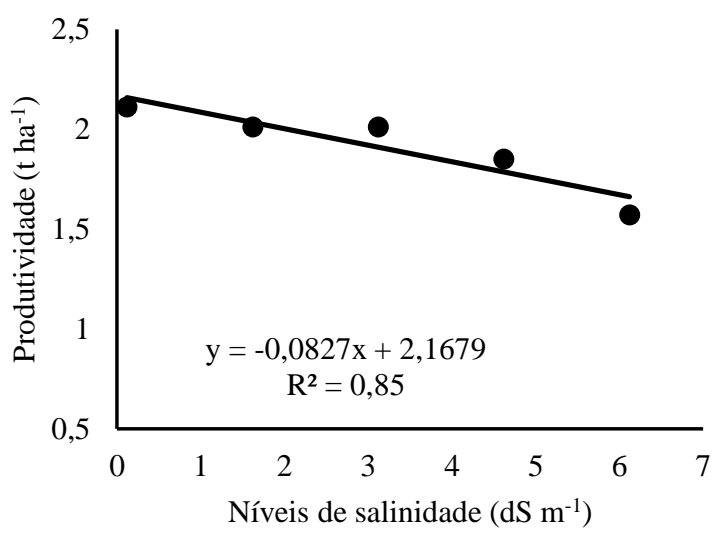

Figura 5. Produtividade $\left(\mathrm{t} \mathrm{ha} \mathrm{h}^{-1}\right) \mathrm{da}$ couve folha em função de níveis de salinidade $\left(\mathrm{dS} \mathrm{m}^{-1}\right)$. 
Neste sentido, Jamil et al. (2007), verificaram que para a couve-folha a salinidade teve efeito considerável no número de folhas por planta e também sobre a área foliar, além da massa fresca da parte aérea e massa das raízes terem sido fortemente inibidos devido os níveis de salinidade, o que reduziu consideravelmente a produtividade da cultura.

Neste sentido, Oliveira et al. (2012) estudando a cultura da rúcula, encontraram uma redução linear dos parâmetros produtivos de folhosas, por aumento unitário da salinidade. Esse efeito é devido à resposta das plantas à salinidade, mediante sua capacidade de adaptação osmótica pela diminuição do teor de água nas folhas ou pelo aumento da concentração de solutos (DIAS et al., 2011).

\section{CONCLUSÕES}

A salinidade reduz o crescimento e a produtividade da couve folha.

A aplicação exógena de ácido ascórbico aumenta o diâmetro da copa da couve folha.

\section{REFERÊNCIAS}

AKRAM, Nudrat A.; SHAFIQ, Fahad; ASHRAF, Muhammad. Ascorbic acid-a potential oxidant scavenger and its role in plant development and abiotic stress tolerance. Frontiers in plant science, v. 8, p. 613, 2017.

ALVES, R. C.; MEDEIROS, A. S.; NICOLAU, M. C. M.; PIZOLATO NETO, A.; OLIVEIRA, F. A.; LIMA, L. W.; TEZOTO, T.; GRATÃO, P. L. The partial root-zone saline irrigation system and antioxidant responses in tomato plants. Plant Phisiology and Biochemistry, v.127, n. 1, p. 366-379, 2018.

ATHAR, H.; KHAN, A.; ASHRAF, M. Exogenously applied ascorbic acid alleviates salt-induced oxidative stress in wheat. Environmental and Experimental Botany, v. 63, n. 1, p. 224-231, 2008.
DIAS, M. S.; REIS, L. S.; SANTOS, R. H. S.; ALMEIDA, C. A. C.; PAES, R. A.; ALBUQUERQUE, A. W.; SILVA, F. A. Colloquium Agrariae, v. 15, n.4, p. 22-30, 2019.

DIAS, N. S.; BLANCO, F. F. Efeitos dos sais no solo e na planta. In: GHEYI, H. R.; DIAS, N. S.; LACERDA, C. F. (Ed.). Manejo da salinidade na agricultura: estudos básicos e aplicados. Fortaleza: INCT Sal, 2011. cap.9, p. 129-141.

DOS SANTOS, O. F., BROETTO, F., DE OLIVEIRA, D. P. F., GALVÃO, Í. M., DE SOUZA, M. L. C., \& BASÍlIO, J. J. N.. Ácido ascórbico, uma alternativa para minimizar os efeitos da deficiência hídrica em rabanete. IRRIGA, v. 1, n.1, p. 79-91, 2018.

EMBRAPA- Empresa Brasileira de Pesquisa Agropecuária. Centro Nacional de Pesquisa de Solos. 3. ed. Brasília: EMBRAPA-SPI, 2018. 353 p.

EMPRESA BRASILEIRA DE PESQUISA AGROPECUÁRIA. Centro Nacional de Pesquisa de Solos. Sistema Brasileiro de Classificação de Solos. 3. Ed. Rio de Janeiro, 2013. 306p.

FERREIRA, D. F. Sisvar: a computer statistical analysis system. Ciência e Agrotecnologia, v.35, p.1039-1042, 2011.

FILGUEIRA, F. A. R. Novo manual de olericultura: agrotecnologia moderna na produção e comercialização de hortaliças. Viçosa: Editora UVF, p. 421. 2013.

GARCIA， G. O.; NAZÁRIO, A. A.; MORAES, W. B.; GONÇALVES, I. Z.; MADALÃO, J. C. Respostas de genótipos de feijoeiro a salinidade. Engenharia na Agricultura, v.18, n.4, p.330-338, 2010.

GALLIE, D. R., "L-Ascorbic Acid: A Multifunctional Molecule Supporting Plant Growth and Development", Scientifica, vol. 2013, p. 24, 2013. 
HAMEED, A. GULZAR， S.; AZIZ， I.; HUSSAIN, T.; GUL, B. Effects of salinity and ascorbic acid on growth, water status and antioxidant system in a perennial halophyte. AoB Plants, v. 7, n. 4, p. 1-11, 2015.

JAMIL, M.; REHMAN, S.; RHA, E.S. Salinity effect on plant growth, PSII photochemistry and chlorophyll content in sugar beet (Beta vulgaris L.) and cabbage (Brassica oleracea capitate L.). Pakistan Journal of Botany, v.39, p.753 - 760, 2007.

Köppen, W. 1948. Climatologia: con un estudio de los climas de la tierra. Fondo de Cultura Econômica. México. 479p.

LIMA JÚNIOR, L. A.; OLIVEIRA, F. S.; ALVES, R. C.; LINHARES, P. S. F.; MEDEIROS, A. M. A.; et al. Tolerância da berinjela à salinidade da água de irrigação. Revista Agroambiente, v. 9, n. 1, p. 27-34, 2015.

LÚCIO, J. C. B.; ALVES, R. C.; OLIVEIRA, K. R.; SILVA, J. S.; PRADO, E. R.; PRADO, E. R.; CARREGA, W. C. Ácido ascórbico na atenuação do estresse salino em amendoinzeiro. XV Encontro Sobre a Cultura do Amendoim. Centro de convenções da FCAV/UNESP - Câmpus de Jaboticabal, SP, 2018.

MAHAJAN, S.; TUTEJA, N. Cold, salinity and drought stresses: An overview. Archives of Biochemistry and Biophysics, v.444, p.139-158, 2005.

MUKHTAR, A.; AKRAM, N. A.; ASHRAF, M. Foliar-applied ascorbic acid enhances antioxidative potential and drought tolerance in cauliflower (Brassica oleracea L. var. Botrytis). Agrochimica, v. 60 p. 100-113, 2016.

OLIVEIRA, A. M. P.; OLIVEIRA, A. M.; DIAS, N. S.; FREITAS, M. Cultivo de rabanete irrigado com água salina. Revista Verde de Agroecologia e Desenvolvimento Sustentável, V.7, p. 01-05, 2012.
OZGUR R, UZILDAY B.; SEKMEN AH. Reactive oxygen species regulation and antioxidant defence in halophytes. Functional Plant Biology,v. 40, n. 1, p.832-847, 2013.

PAIVA, F. I. G. Manejo da fertirrigação potássica e cálcica na cultura do tomateiro cultivadas em ambiente protegido e submetidas ao estresse salino. Dissertação de Mestrado. programa de Pós-graduação em Manejo de Solo e Água da Universidade Federal Rural do Semiárido, Brasil, 2017.

PEREIRA, E. M.; LEITE, D. D. F.; FIDELIS, V. R. L.; PORTO, R. M.; OLIVEIRA, M. I. V. Caracterização físicoquímica de hortaliças tipo folha comercializadas no Brejo Paraibano. Revista agropecuária técnica, v. 37 , n. 1, p. 19-22, 2016.

RICHARDS, L.A. Diagnosis and improvement of saline and alkali soils. Washington: US Department of Agriculture, 1954. 160p.

SILVA, A. L.; NASCIMENTO, M. N.; TANAN, T.T.; OLIVEIRA, U. C. Efeito da salinidade da água de irrigação na produção de alface crespa. Enciclopédia Biosfera, v. 14, n. 26, p. 328, 2017.

SILVA, F. L. B.; LACERDA, C. F.; NEVES, A. L. R.; SOUSA, G. G.; SOUSA, C. H. C. Irrigação com águas salinas e uso de biofertilizante bovino nas trocas gasosas e produtividade de feijão de corda. Irriga, v. 8, n. 2, p. 3014317, 2013.

VIANA, P. C. Eficiência do uso de águas salobras no cultivo hidropônico da couvefolha / Paula Carneiro Viana. Cruz das Almas, BA, 2017.

XAVIER, R. A; DORNELlAS, P. C. Análise do comportamento das chuvas no município de Arapiraca, região Agreste de Alagoas. GEOGRAFIA, Londrina, v. 14, n. 2, p. 49-64, 2010. 